\title{
CONSUMO DE ULTRAPROCESSADOS E ESTADO NUTRICIONAL DE CRIANÇAS COM TRANSTORNO DO ESPECTRO DO AUTISMO
}

\author{
Consumption of ultra-processed foods and nutritional status of children with \\ autism spectrum disorder
}

\section{Consumo de ultraprocesados y estado nutricional de niños con trastorno del espectro autista}

\author{
Ana Karla de Araújo Almeida \\ Universidade CEUMA - São Luís - (MA) - Brasil \\ Poliana Cristina de Almeida Fonseca (Lattes) \\ Universidade Federal do Maranhão - UFMA - São Luís - (MA) - Brasil \\ Leila Alves Oliveira (Lattes) \\ Universidade CEUMA - São Luís - (MA) - Brasil \\ Wyllyane Rayana Chaves Carvalho Santos \\ Universidade Federal do Maranhão - UFMA - São Luís - (MA) - Brasil
}

\section{Adrielle Zagmignan (OrcID)}

Universidade CEUMA - São Luís - (MA) - Brasil

Bianca Rodrigues de Oliveira (OrcID)

Universidade Federal do Maranhão - UFMA - São Luís - (MA) - Brasil

Virgínia Nunes Lima (Lattes)

Universidade CEUMA - São Luís - (MA) - Brasil

Carolina Abreu de Carvalho (Lattes)

Instituto Federal do Maranhão - IFMA - São Luís - (MA) - Brasil

\section{RESUMO}

Objetivo: Analisar o consumo de alimentos ultraprocessados entre crianças com transtorno do espectro do autismo (TEA) e sua associação com o estado nutricional. Métodos: Realizou-se um estudo transversal, em São Luís, Maranhão, Brasil, com amostra de 29 crianças, em 2017. Utilizou-se um questionário semiestruturado, aplicado aos pais ou responsáveis, para obtenção de variáveis sociodemográficas. O estado nutricional foi avaliado pelos indicadores de índice de massa corporal/idade e estatura/ idade. Obteve-se o consumo alimentar por meio de recordatório de $24 \mathrm{~h}$, a partir do qual foi calculado o percentual de contribuição calórica e a média dos alimentos consumidos de acordo com o nível de processamento. Para comparação do consumo dos ultraprocessados de acordo com o estado nutricional, utilizou-se o teste t de Student, com nível de significância de 5\%. Resultados: Verificou-se o excesso de peso em $55,2 \%(n=16)$ das crianças e o consumo de alimentos ultraprocessados foi responsável por $28 \%$ (560 kcal/dia) da contribuição calórica. Crianças com excesso de peso consumiram maior média percentual de alimentos ultraprocessados do que as sem excesso de peso $(34,2 \%$ versus $19,4 \%, p=0,009)$. O consumo de frutas representou apenas $4,3 \%$ (74,6 kcal) da contribuição calórica total, e as hortaliças foram os alimentos in natura menos consumidos pelas crianças. Conclusão: Alimentos in natura ou minimamente processados foram a base da alimentação das crianças estudadas. Apesar disso, o maior consumo de alimentos ultraprocessados foi associado ao excesso de peso nas crianças com TEA.

Descritores: Transtorno Autístico; Consumo de Alimentos; Estado Nutricional; Criança.

\section{ABSTRACT}

Objective: To analyze the consumption of ultra-processed foods among children with autism spectrum disorder (ASD) and its association with nutritional status. Methods: Cross-sectional study of 29 children conducted in 2017 in São Luís, Maranhão, Brazil. A semistructured questionnaire was used with parents or caregivers to obtain sociodemographic information. The nutritional status 
was assessed based on body mass index/age and height/age. The food consumption was assessed using a 24h recall, which was used to estimate the percentage of caloric contribution and the mean of the foods consumed according to the level of processing. Student's $t$ test with a significance level of $5 \%$ was used to compare the consumption of ultra-processed foods in relation to the nutritional status. Results: Overweight was observed in $55.2 \%(n=16)$ of the children and the consumption of ultra-processed foods was responsible for $28 \%$ (560 kcal/day) of caloric contribution. Overweight children consumed a higher mean percentage of ultra-processed foods than those who were not overweight $(34.2 \%$ vs. $19.4 \%, p=0.009)$. Fruit consumption represented only $4.3 \%$ (74.6 kcal) of total caloric contribution and vegetables were the least consumed whole foods among children. Conclusion: Whole foods or minimally processed foods were the most consumed food among the analyzed children. However, the higher consumption of ultra-processed foods was associated with overweight in children with ASD.

Descriptors: Autistic Disorder; Food Consumption; Nutritional Status; Children.

\section{RESUMEN}

Objetivo: Analizar el consumo de alimentos ultraprocesados entre los niños con trastorno del espectro autista (TEA) y su asociación con el estado nutricional. Métodos: Se realizó un estudio transversal, en São Luís, Maranhão, Brasil, con una muestra de 29 niños en 2017. Se utilizó un cuestionario semi-estructurado que ha sido aplicado a los padres o responsables para obtener las variables sociodemográficas. El estado nutricional ha sido evaluado por los indicadores del índice de masa corporal/edad y estatura/edad. Se obtuvo el consumo alimentario a través del recordatorio de 24 horas del cual ha sido calculado el porcentaje de la contribución calórica y la media de los alimentos consumidos según el nivel de procesamiento. Para comparar el consumo de los ultraprocesados según el estado nutricional se utilizó la prueba t de Student con el nivel de significación del 5\%. Resultados: Se verificó el exceso de peso en el 55,2\% $(n=16)$ de los niños y el consumo de alimentos ultraprocesados ha sido responsable por el 28\% (560 kcal/día) de la contribución calórica. Niños con exceso de peso han consumido la mayor media percentual de alimentos ultraprocesados que los sin el exceso de peso (34,2\% versus 19,4\%, p=0,009). El consumo de frutas ha representado solamente el 4,3\% (74,6 kcal) de la contribución calórica total y las hortalizas han sido los alimentos in natura menos consumidos por los niños. Conclusión: Alimentos in natura o los muy poco procesados fueron la base de la alimentación de los niños estudiados. Sin embargo, el mayor consumo de alimentos ultraprocesados se asoció con el exceso de peso de los niños con TEA.

Descriptores: Trastorno Autístico; Consumo de Alimentos; Estado Nutricional; Niño.

\section{INTRODUÇÃO}

O Transtorno do Espectro do Autismo (TEA) é caracterizado por um conjunto de alterações atípicas no neurodesenvolvimento da criança. Crianças portadoras do TEA apresentam dificuldades de interação social, atrasos cognitivos na linguagem e deficiências na comunicação, além de comportamentos repetitivos e restritivos ${ }^{(1)}$.

Nos últimos anos, a prevalência de TEA apresentou aumento significativo. Estimativas mais recentes do Centers for Disease Control and Prevention (CDC), com base em dados obtidos em 2014, nos Estados Unidos, revelam uma prevalência de 16,8 por 1000 , ou seja, uma em cada 59 crianças com oito anos de idade tem TEA(2).

Além das características percebidas no comportamento, na linguagem e na interação social, as comorbidades gastrointestinais são comuns em crianças com TEA, tais como: constipação crônica, diarreia e dor abdominal. E, apesar de muitas vezes negligenciadas, há evidência da associação entre a presença de sintomas gastroinstestinais e a gravidade da síndrome, uma vez que indivíduos que cursam com o TEA e sintomas gastrointestinais tendem a apresentar maior irritabilidade, ansiedade e isolamento social ${ }^{(3)}$.

Com relação ao hábito alimentar dessas crianças, a seletividade alimentar é a preocupação mais comum, em virtude da sua repercussão negativa no estado nutricional e no crescimento(4). Portanto, deve-se ter atenção à ingestão de alimentos não saudáveis, bem como à restrição e monotonia alimentar dessas crianças. Devido a essas peculiaridades no comportamento alimentar, crianças com TEA tendem a ter algumas deficiências nutricionais. As deficiências mais comuns nesse grupo são as de cálcio, zinco, magnésio, antioxidantes e ômega 3, além de excesso de cobre. Por isso, o planejamento e cuidado com a alimentação dessas crianças devem ser redobrados, a fim de garantir-lhes uma boa nutrição( ${ }^{(5)}$.

Atualmente, tem sido muito discutida a elevada participação dos alimentos ultraprocessados na dieta infantil. Um estudo transversal ${ }^{(6)}$ realizado em uma Unidade Básica de Saúde no Sul do Brasil, com 204 crianças de 2 a 10 anos, revelou que a contribuição calórica dos ultraprocessados na dieta infantil é elevada. O consumo médio de energia foi de $1.672,3 \mathrm{kcal} / \mathrm{dia}$, sendo $47 \%$ dessas calorias provenientes de alimentos ultraprocessados. 
Outros estudos também têm apontado a elevada contribuição calórica dos alimentos ultraprocessados na alimentação infantil( ${ }^{(7-9)}$. Sabe-se que os alimentos ultraprocessados apresentam alta densidade energética, excesso de gorduras totais e saturadas, baixo teor de fibras e maior concentração de açúcar e sódio, além de apresentarem em sua composição uma quantidade deficiente de vitaminas e minerais, essenciais para o controle das deficiências nutricionais apresentadas pelos autistas ${ }^{(10,11)}$.

O consumo de alimentos ultraprocessados vem sendo associado ao excesso de peso em adolescentes e adultos sem TEA ${ }^{(12-14)}$. Essa associação é explicada pela baixa qualidade nutricional dos alimentos ultraprocessados. Até o momento, não localizamos estudos publicados sobre o consumo de alimentos de acordo com o propósito e extensão do processamento em crianças com TEA, bem como estudos que tenham associado esse consumo ao estado nutricional.

Diante da natureza complexa do TEA e considerando o maior risco dessas crianças desenvolverem desvios nutricionais, a avaliação do perfil dos alimentos consumidos por elas, bem como sua associação ao estado nutricional, constitui-se em uma ferramenta de diagnóstico e prevenção de agravos a saúde. O que, por sua vez, oportuniza a implementação de estratégias multidisciplinares específicas, a fim de promover atenção integral à saúde dessa população. Portanto, o objetivo deste estudo foi analisar o consumo de alimentos ultraprocessados entre crianças com transtorno do espectro autista e sua associação ao estado nutricional.

\section{MÉTODOS}

Realizou-se um estudo transversal e descritivo, cuja coleta de dados ocorreu no período de maio a setembro de 2017, em São Luís, Maranhão, Brasil, com crianças diagnosticadas com transtorno do espectro autista (TEA).

Incluíram-se, de forma não probabilística, em uma amostra por conveniência, 29 crianças, de idades entre $3 \mathrm{e}$ 12 anos, diagnosticadas com TEA por um neuropediatra. Realizou-se a coleta de dados a partir de entrevista, com os pais ou responsáveis de cada criança, na Clínica Escola de uma universidade particular de São Luís.

Utilizou-se um questionário semiestruturado, elaborado pelos pesquisadores, com perguntas relacionadas a condições socioeconômicas (renda familiar mensal, escolaridade do responsável, estado civil e ocupação) e questões sobre seletividade alimentar ou dificuldade das crianças em aceitar novos alimentos.

Os dados antropométricos foram obtidos através da aferição do peso e altura das crianças, de acordo com as técnicas preconizadas pela Organização Mundial da Saúde ${ }^{(15)}$. Para a aferição da altura (em centímetros), utilizou-se um estadiômetro, com capacidade máxima de $215 \mathrm{~cm}$, acoplado a uma balança digital da marca Filizola ${ }^{\circledR}$. O peso foi aferido com essa balança digital, com capacidade de duzentos quilos e cinquenta gramas de precisão. Após a aferição, calculou-se o índice de massa corporal (IMC) por meio da fórmula IMC = peso $(\mathrm{kg}) /$ altura $\left(\mathrm{m}^{2}\right)$.

Para o diagnóstico nutricional, utilizaram-se o escore $z$, do IMC, para a idade e estatura para a idade, de acordo com os valores de referência proposto pela Organização Mundial de Saúde ${ }^{(15)}$.

As informações sobre o consumo alimentar foram obtidas por meio de um recordatório de 24 horas (R24h), aplicado por um pesquisador devidamente treinado. Aplicou-se um único R24h, em referência ao consumo alimentar da criança no dia anterior. Foram registrados todos os alimentos e bebidas ingeridos pela criança, com detalhes de preparação, quantidades e marcas. Posteriormente, classificaram-se os alimentos de acordo com o nível de processamento, segundo o Guia Alimentar da População Brasileira( ${ }^{(16)}$, cuja classificação agrupa os alimentos em três categorias: alimentos in natura ou minimamente processados, alimentos processados e alimentos ultraprocessados.

$\mathrm{Na}$ categoria de alimentos in natura ou minimamente processados estão aqueles obtidos diretamente de plantas ou de animais, que não sofrem qualquer alteração após deixar a natureza. Os alimentos minimamente processados correspondem a alimentos in natura que foram submetidos a processos de limpeza, remoção de partes não comestíveis ou indesejáveis. Entre os alimentos processados estão incluídos produtos fabricados pela indústria essencialmente com a adição de sal ou açúcar, ou outra substância de uso culinário, a alimentos in natura para torná-los duráveis e mais agradáveis ao paladar.

Na categoria de alimentos ultraprocessados estão formulações industriais feitas, inteiramente ou majoritariamente, de substâncias extraídas de alimentos, derivadas de constituintes de alimentos, ou sintetizadas em laboratório com base em matérias orgânicas, como corantes, aromatizantes, realçadores de sabor e outros tipos de aditivo usados para dotar os produtos de propriedades sensoriais.

O R24h foi analisado no software AvaNutri ${ }^{\circledR}$, versão Revolution (Rio de Janeiro, Brasil), para o cálculo da quantidade de calorias fornecidas pelos alimentos. Posteriormente, exportaram-se os dados para o software Stata ${ }^{\circledR}$, versão 14.0 (College Station, Texas, USA), no qual calculou-se o percentual de contribuição calórica e a média dos 
alimentos consumidos de acordo com o nível de processamento. Para caracterização da amostra, calcularam-se os percentuais de variáveis referentes à situação socioeconômica, ao estado nutricional e ao comportamento alimentar.

Para a análise da associação entre a média de consumo de alimentos ultraprocessados e o excesso de peso, utilizou-se o teste t de Student, com nível de significância fixado em $5 \%$.

O presente estudo recebeu aprovação do Comitê de Ética em Pesquisa da Associação de Ensino Superior CEUMA, sob Parecer $n^{\circ}$ 2.042.597. Obteve-se a autorização para a participação das crianças na pesquisa por meio da assinatura do Termo de Consentimento Livre e Esclarecido pelos pais ou responsáveis.

\section{RESULTADOS}

Dentre os pais ou responsáveis entrevistados, verificou-se: idade média de 37 anos; a maioria com renda mensal de um ou mais de um salário mínimo $(89,7 \% ; n=26)$, e ensino médio incompleto ou completo $(72,5 \% ; n=21)$. As crianças apresentaram média de 8 anos e $96,6 \%(n=28)$ eram do sexo masculino. Observou-se que $69,0 \%(n=20)$ das crianças com TEA apresentaram seletividade alimentar e dificuldades para aceitar novos alimentos (Tabela I).

$\mathrm{Na}$ Tabela II, destaca-se o alto percentual de crianças autistas que apresentaram excesso de peso $(55,2 \%$; $n=16)$. Ademais, a prevalência de baixo peso $(20,7 \% ; n=6)$ e baixa estatura $(13,8 \% ; n=4)$ também foram elevadas.

Tabela I - Características gerais dos pais ou responsáveis e das crianças com TEA. São Luís, Maranhão, 2017.

\begin{tabular}{|c|c|c|}
\hline Variáveis & $\mathbf{n}$ & $\%$ \\
\hline \multicolumn{3}{|l|}{ Idade do entrevistado } \\
\hline 20-34 anos & 7 & 24,1 \\
\hline$>35$ anos & 22 & 75,9 \\
\hline \multicolumn{3}{|l|}{ Grau de parentesco do entrevistado } \\
\hline Mãe ou pai & 28 & 96,6 \\
\hline Avó ou outro familiar & 1 & 3,4 \\
\hline \multicolumn{3}{|l|}{ Renda mensal } \\
\hline < 1 Salário mínimo & 3 & 10,3 \\
\hline 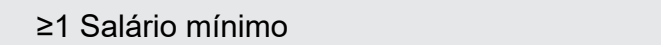 & 26 & 89,7 \\
\hline \multicolumn{3}{|l|}{ Escolaridade do entrevistado } \\
\hline Ensino fundamental incompleto ou completo & 3 & 10,3 \\
\hline Ensino médio incompleto ou completo & 21 & 72,5 \\
\hline Ensino superior incompleto ou completo & 5 & 17,2 \\
\hline \multicolumn{3}{|l|}{ Sexo da criança } \\
\hline Feminino & 1 & 3,4 \\
\hline Masculino & 28 & 96,6 \\
\hline \multicolumn{3}{|l|}{ Idade da criança } \\
\hline$<5$ anos & 4 & 13,8 \\
\hline 5 a 12 anos & 25 & 86,2 \\
\hline \multicolumn{3}{|c|}{ Seletividade alimentar e/ou dificuldade em aceitar novos alimentos } \\
\hline $\operatorname{Sim}$ & 20 & 69,0 \\
\hline Não & 9 & 31,0 \\
\hline TOTAL & 29 & 100 \\
\hline
\end{tabular}

Tabela II - Estado nutricional de crianças com transtorno do espectro do autismo de acordo com o indicador índice de massa corporal para idade (IMC/I) e estatura para idade (E/I). São Luís, Maranhão, 2017.

\begin{tabular}{lcc}
\hline Variáveis & $\%$ & $\mathbf{n}$ \\
\hline Índice de massa corporal para idade (IMC/I) & & 6 \\
Baixo peso & 20,7 & 7 \\
Adequado & 24,1 & 10 \\
Sobrepeso & 34,5 & 6 \\
Obesidade & 20,7 & \multicolumn{2}{c}{4} \\
Estatura para idade (E/I) & & 24 \\
Baixa estatura & 13,8 & 2 \\
Adequada & 82,8 & 1 \\
Elevada & 3,4 & \\
\hline
\end{tabular}


A maior contribuição calórica observada foi proveniente dos alimentos in natura ou minimamente processados, com $61,5 \%$ (1249,7 kcal/dia). O consumo de alimentos ultraprocessados representou $27,6 \%$ da contribuição calórica, equivalente a uma média de 560,0 kcal por dia (Tabela III).

Os alimentos in natura com maior contribuição calórica compreenderam a carne branca e os ovos, representando $15,5 \%$ (326,8 kcal/dia), seguidos pelo arroz, que correspondeu a 12,1\% (264 kcal/dia) do percentual de consumo total de calorias. O consumo de frutas representou apenas 4,3\% (74,6 kcal) da contribuição calórica total. As hortaliças representaram os alimentos in natura ou minimamente processados menos consumidos pelas crianças, com percentual correspondente a apenas $0,3 \%$ (7,3 kcal/dia) da contribuição calórica total (Tabela IV).

A maior contribuição calórica dos alimentos processados originou-se do pão francês, com média de 125,2 kcal/ dia, representando $6,3 \%$ do consumo total de calorias. E o alimento processado com menor contribuição calórica foi a sardinha enlatada $(0,2 \%)$ (Tabela IV).

Dentre os alimentos ultraprocessados, os biscoitos (salgado e doce) foram responsáveis pelo maior aporte calórico, representando $13,5 \%$ (247,7 kcal/dia). A farinha para mingau foi o segundo alimento ultraprocessado mais consumido, apresentando uma média de $112,4 \mathrm{kcal} / \mathrm{dia}$, correspondendo a $4,6 \%$ do valor calórico total. $O$ alimento ultraprocessado menos consumido foi a margarina, com média de $7,5 \mathrm{kcal} / \mathrm{dia}$, representando $0,4 \%$ do valor calórico total (Tabela IV).

$\mathrm{Na}$ análise da associação entre a média de consumo de alimentos ultraprocessados e o excesso de peso, observouse que crianças sem excesso de peso tinham a média de 19,4\% de participação de alimentos ultraprocessados, enquanto as crianças com excesso de peso apresentaram $34,2 \%(p=0,009)$.

Tabela III - Média e percentual de calorias dos grupos de alimentos in natura ou minimamente processados, processados e ultraprocessados por crianças com transtorno do espectro do autismo. São Luís, Maranhão, 2017.

\begin{tabular}{lcc}
\hline Grupos alimentares & Média \pm DP & Consumo do VET (\%) \\
\hline Alimentos in natura ou minimamente processados & $1249,7 \pm 517,0$ & 61,5 \\
Alimentos processados & $140,6 \pm 149,2$ & 7,1 \\
Alimentos ultraprocessados & $560,0 \pm 357,1$ & 27,6 \\
Total & 1950,3 & 100,0 \\
\hline
\end{tabular}

DP: Desvio Padrão; VET: Valor energético total

Tabela IV - Média e percentual de consumo de alimentos in natura ou minimamente processados, alimentos processados e alimentos ultraprocessados por crianças com transtorno do espectro do autismo. São Luís, Maranhão, 2017.

\begin{tabular}{|c|c|c|}
\hline Variavéis & Média & Consumo do VET(\%) \\
\hline \multicolumn{3}{|l|}{ Alimentos in natura ou minimamente processados } \\
\hline Hortaliças & 7,2 & 0,3 \\
\hline Farinha Torrada & 17,6 & 1,0 \\
\hline Café (adoçado) & 26,0 & 1,4 \\
\hline Sopa de Legumes & 30,3 & 2,1 \\
\hline Macarrão & 72,8 & 3,2 \\
\hline Feijões & 78,6 & 3,8 \\
\hline Carne Vermelha & 75,5 & 3,9 \\
\hline Frutas (Maçã, Melancia, Banana, Laranja e Açaí) & 74,6 & 4,3 \\
\hline Sucos de Frutas & 99,7 & 4,5 \\
\hline Leite & 207,3 & 9,4 \\
\hline Arroz & 264,0 & 12,1 \\
\hline Carne Branca e Ovos & 326,8 & 15,5 \\
\hline \multicolumn{3}{|l|}{ Alimentos Processados } \\
\hline Sardinha & 2,8 & 0,2 \\
\hline Queijo & 12,6 & 0,6 \\
\hline Pão Francês & 125,2 & 6,3 \\
\hline \multicolumn{3}{|l|}{ Alimentos Ultraprocessados } \\
\hline Margarina & 7,5 & 0,4 \\
\hline logurte & 23,2 & 1,0 \\
\hline Suco Artificial & 31,0 & 1,5 \\
\hline Fast food (Pizza e Coxinha) & 31,3 & 1,5 \\
\hline Pães (Formas e Doce) & 56,0 & 3,0 \\
\hline Farinhas para Mingau & 112,4 & 4,6 \\
\hline Biscoitos (Doce e Salgado) & 247,7 & 13,5 \\
\hline
\end{tabular}

VET: Valor energético total 


\section{DISCUSSÃO}

Alimentos in natura ou minimamente processados representaram $61 \%$ do total de calorias ingeridas, sendo, portanto, a base da alimentação das crianças com TEA estudadas na presente pesquisa. Apesar disso, o maior consumo de alimentos ultraprocessados associou-se com a presença de excesso de peso. O consumo de alimentos ultraprocessados correspondeu a $27,6 \%$ do consumo total, percentual que não pode ser considerado baixo, uma vez que o consumo desses alimentos não é recomendado pelo Guia Alimentar para a População Brasileira( ${ }^{(16)}$. Ademais, a frequência de desvios nutricionais foi elevada entre as crianças com TEA, sendo esse um achado preocupante do atual estudo.

O elevado consumo de alimentos ultraprocessados entre adolescentes tem sido associado à síndrome metabólica ${ }^{(14)}$. Estudos realizados com jovens e adultos no Brasil também encontraram associação entre o consumo de alimentos ultraprocessados e excesso de peso ${ }^{(12,13)}$. Mesmo não havendo estudos em crianças com TEA que tenham investigado essa associação, as recomendações do Guia Alimentar para a População Brasileira, destacam que os alimentos ultraprocessados são nutricionalmente desbalanceados e contribuem para o desenvolvimento de excessos ou déficits de nutrientes ${ }^{(16)}$, concordando com os resultados do presente estudo.

O percentual de consumo de alimentos in natura ou minimamente processados, neste estudo, pode ser considerado positivo, entretanto, preocupa a constatação de que apenas uma pequena parte desse consumo é oriunda de frutas, legumes e verduras. Em um estudo comparativo, realizado com crianças com TEA e sem TEA atendidas em um hospital no Norte da Índia, observou-se que as crianças com TEA apresentaram aversão a frutas e vegetais ${ }^{(17)}$. Estudos apontam que as crianças com TEA têm preferências por alimentos a base de amido, lanches e alimentos ultraprocessados, e apresentam relutância ao consumo de frutas e vegetais ${ }^{(18-20)}$.

Sabe-se que frutas e hortaliças são alimentos de grande importância para o aporte de micronutrientes essenciais ao crescimento e desenvolvimento da criança, além de auxiliarem na prevenção e no controle de doenças e reforçarem o sistema imune ${ }^{(21)}$. Há uma correlação significante entre carências de micronutrientes e a piora dos sintomas autísticos ${ }^{(20)}$. Portanto, o baixo consumo de frutas e hortaliças representa um risco para a saúde das crianças com TEA, pois pode levar a carências nutricionais e afetar negativamente a sintomatologia da doença.

Estudo realizado com crianças com TEA em Curitiba, Paraná, apontou que as crianças estudadas tinham uma alta frequência de consumo de alimentos ultraprocessados como embutidos, refrigerantes, sucos artificiais e doces, sendo que o suco artificial era consumido duas ou mais vezes ao dia(22). $^{(2)}$

Os alimentos ultraprocessados mais consumidos pelas crianças estudadas foram os biscoitos salgados e doces, seguidos pela farinha para mingau. Um estudo, realizado com os dados secundários da Pesquisa Nacional de Demografia e Saúde, demonstrou que o consumo de biscoitos e bolachas pela população infantil é elevado. Cerca de $50 \%$ das crianças, com idade de 6 a 59 meses, de todas as regiões (exceto a Região Norte), consumiam esses alimentos diariamente ${ }^{(23)}$. Outro estudo realizado entre crianças com TEA e crianças com desenvolvimento típico, com idade de 7 a 10 anos, demonstrou que $63 \%$ das crianças com TEA eram seletivas na textura dos alimentos, tendo preferência por alimentos crocantes, como pizza, biscoitos e doces ${ }^{(24)}$.

Estudos que avaliaram o consumo de alimentos ultraprocessados em crianças com o desenvolvimento típico constataram uma contribuição calórica de alimentos ultraprocessados maior que a do presente estudo. Em uma investigação sobre o consumo alimentar de crianças com idade entre 2 e 10 anos, encontrou-se a contribuição calórica de $47 \%{ }^{(6)}$. Outra pesquisa, realizada com crianças com idade de 7 e 8 anos, mostrou uma contribuição calórica de $48,6 \%$ de alimentos processados e ultraprocessados ${ }^{(25)}$. No presente estudo, a contribuição calórica oriunda dos alimentos ultraprocessados foi de $27,6 \%$, portanto, inferior aos estudos relatados.

A avaliação do estado nutricional das crianças com TEA evidenciou uma dupla carga de desvios nutricionais, que sugerem o consumo de uma alimentação desequilibrada por essas crianças. Contudo, ao mesmo tempo em que a prevalência de excesso de peso entre essas crianças foi elevada, atingindo mais da metade da amostra, o percentual de crianças com baixo peso $(20,7 \%)$ e baixa estatura para idade $(13,8 \%)$ também foi significativo.

Este fato é preocupante, pois o excesso de peso na população infantil tem aumentado e está relacionado ao desenvolvimento de doenças crônicas não transmissíveis na vida adulta ${ }^{(26)}$. Ademais, a exposição à desnutrição pode trazer sérias consequências à saúde da criança, impossibilitando o crescimento e desenvolvimento normal, e comprometendo sua imunidade, além de interferir em sua qualidade de vida ${ }^{(21)}$. Seletividade alimentar e rejeição a novos alimentos foram problemas alimentares com alta prevalência na amostra deste estudo. Estas podem afetar a ingestão de nutrientes e calorias e, possivelmente, estar na gênese dos desvios nutricionais observados no presente estudo ${ }^{(27)}$. 
Em um estudo comparativo de coorte, realizado nos Estados Unidos, com crianças com TEA e um grupo controle de crianças com desenvolvimento típico, foi observado que as crianças com TEA de 2 a 5 anos eram mais propensas ao excesso de peso. Por outro lado, entre as crianças de 6 a 11 anos, a prevalência de baixo peso foi maior nas crianças com TEA do que no grupo controle. Esses resultados também concordam com a dupla carga de desvios nutricionais observados na presente pesquisa. De acordo com os autores do estudo americano, esse resultado pode ser explicado pelo fato de crianças com idade de 2 a 5 anos passarem mais tempo em tratamento terapêutico, em que lanches são usados para contribuir com a participação da criança, e também por terem menos oportunidades de praticar atividades físicas. Entre as crianças com TEA de 6 a 11 anos, a alta prevalência de baixo peso foi justificada pela autonomia nas escolhas e acesso aos alimentos nessa idade ${ }^{(28)}$.

Os valores encontrados na atual pesquisa relativos ao excesso de peso em crianças com TEA são semelhantes aos relatados em um estudo realizado com crianças com TEA de 2 a 6 anos, em Curitiba, Paraná, no qual encontrou-se a frequência de excesso de peso de $41,2 \%{ }^{(22)}$. Percentuais elevados de excesso de peso também foram encontrados em crianças de 3 a 10 anos de idade, diagnosticadas com TEA, no município de Limoeiro do Norte, Ceará. Os resultados demonstraram elevados índices de sobrepeso $(23,1 \%)$ e de obesidade $(15,38 \%)^{(29)}$. Por outro lado, um estudo conduzido nos centros estaduais de Cartum, no Sudão, mostrou frequências menores, com cerca de $20 \%$ das crianças e adolescentes ( 3 a 13 anos) apresentando excesso de peso ${ }^{(30)}$.

A avaliação do consumo alimentar e o diagnóstico nutricional de crianças com TEA contribuem com a vigilância alimentar e nutricional, uma das diretrizes da Política Nacional de Alimentação e Nutrição (PNAN). O objetivo dessa diretriz é o monitoramento da situação alimentar e nutricional com o propósito de melhorar as condições de alimentação da população ${ }^{(31)}$. Além disso, considerando a alimentação como um dos eixos prioritários da Política Nacional de Promoção da Saúde (PNPS), avaliar e identificar como essas crianças estão quanto ao aspecto alimentar e nutricional constituiu-se uma ferramenta importante para a promoção da saúde ${ }^{(32)}$.

Dessa forma, a investigação da presença de distúrbios, bem como a identificação de quais são os alimentos mais consumidos, é uma ponte para a formação e o estabelecimento de estratégias específicas, tendo em vista a proteção e promoção da saúde em todos os níveis da assistência, tanto para as crianças com TEA quanto para seus familiares.

Sabe-se que o TEA exerce forte influência na dinâmica familiar, por isso a necessidade de promover um cuidado integral e longitudinal| ${ }^{(33)}$. No contexto do cuidado e da promoção à saúde, ressalta-se a relevância de incluir o nutricionista na equipe multidisciplinar que atende à criança com TEA, a fim de manejar os problemas alimentares tipicamente desenvolvidos por elas. O nutricionista deve encorajar os pais a incluírem na sua rotina alimentos saudáveis em substituição aos ultraprocessados, os quais, além de estarem associados à presença de excesso de peso, têm impacto significativo na saúde geral da criança $a^{(6)}$. Assim, as estratégias nutricionais devem visar à criação de um vínculo entre a criança e o alimento, principalmente aqueles alimentos que a criança apresenta mais aversão. Ademais, a melhora no consumo alimentar, com a presença de uma alimentação equilibrada e saudável, será capaz de gerar impactos positivos sobre o estado nutricional, o crescimento, o desenvolvimento e os sinas e sintomas próprios da TEA.

O tamanho amostral reduzido e o uso de apenas um R24h podem ser considerados limitações do presente estudo. Entretanto, por tratar-se de um desfecho de baixa frequência em crianças, a obtenção de um grande tamanho amostral é difícil. Além disso, as limitações do uso de apenas um dia de recordatório são atenuadas, uma vez que o consumo alimentar de crianças com TEA tende à monotonia e a um limitado repertório alimentar ${ }^{(34,35)}$.

Destaca-se como ponto forte o ineditismo deste trabalho na avaliação do consumo alimentar de crianças com TEA, a partir da nova classificação dos alimentos, de acordo com a extensão e propósito do processamento e sua associação ao excesso de peso. O diagnóstico do TEA baseado em laudo emitido por neuropediatra como critério de inclusão no estudo é fator que fortalece a qualidade na seleção da amostra.

\section{CONCLUSÃO}

Alimentos in natura ou minimamente processados foram a base da alimentação das crianças estudadas. Apesar disso, o maior consumo de alimentos ultraprocessados esteve associado ao excesso de peso nas crianças com TEA. Portanto, elas e seus familiares devem ser inseridos em programas de educação nutricional para conscientização da importância de uma alimentação adequada e saudável, bem como para a prevenção de agravos nutricionais. 


\section{CONTRIBUIÇÕES}

Ana Karla de Araújo Almeida colaborou na concepção, execução, análise estatística e redação do artigo; Poliana Cristina de Almeida Fonseca, Leila Alves Oliveira e Wyllyane Rayana Chaves Carvalho Santos colaboraram na concepção, análise estatística e revisão final do artigo. Adrielle Zagmignan colaborou na concepção, execução e revisão final do artigo. Bianca Rodrigues de Oliveira colaborou na análise estatística, redação e revisão final do artigo. Virgínia Nunes Lima colaborou na concepção, execução, análise estatística e revisão final do artigo. Carolina Abreu de Carvalho colaborou na concepção, execução, análise estatística, redação e revisão final do artigo.

\section{AGRADECIMENTOS E CONFLITOS DE INTERESSE}

Os autores agradecem aos pais ou responsáveis que concordaram com a participação das crianças no estudo, e declaram não haver conflitos de interesse.

\section{REFERÊNCIAS}

1. Buie T, Campbell DB, Fuchs GJ, Furuta GT, Levy J, Vande Water J, et al. Evaluation, diagnosis, and treatment of gastrointestinal disorders in individuals with asds: a consensus report. pediatrics [Internet]. 2010 Jan [acesso em: 2018 Fev 4];125 Supp 1:S1-18. Disponível em: http://www.ncbi.nlm.nih.gov/pubmed/20048083

2. Baio J, Wiggins L, Christensen DL, Maenner MJ, Daniels J, Warren Z, et al. Prevalence of autism spectrum disorder among children aged 8 years - Autism and Developmental Disabilities Monitoring Network, 11 Sites, United States, 2014. MMWR Surveill Summ [Internet]. 2018 Apr 27 [acesso em 2018 Ago 20];67(6):1-23. Disponível em: http://www.ncbi.nlm.nih.gov/pubmed/29701730

3. Sanctuary MR, Kain JN, Angkustsiri K, German JB. Dietary considerations in autism spectrum disorders: the potential role of protein digestion and microbial putrefaction in the gut-brain axis. Front Nutr [Internet]. 2018 [acesso em 2018 Ago 20];5:40. Disponível em: http://www.ncbi.nlm.nih.gov/pubmed/29868601

4. Barnhill K, Gutierrez A, Ghossainy M, Marediya Z, Devlin M, Sachdev P, et al. Dietary status and nutrient intake of children with autism spectrum disorder: A case-control study. Res Autism Spectr Disord [Internet]. 2018 [acesso em 2018 Ago 20];50:51-9. Disponível em: https://www.sciencedirect.com/science/article/pii/S1750946718300448

5. Won H, Mah W, Kim E. Autism spectrum disorder causes, mechanisms, and treatments: focus on neuronal synapses. Front Mol Neurosci [Internet]. 2013 [acesso em 2017 Dez 29];6:19. Disponível em: http://www.ncbi. nlm.nih.gov/pubmed/23935565

6. Sparrenberger K, Friedrich RR, Schiffner MD, Schuch I, Wagner MB. Ultra-processed food consumption in children from a Basic Health Unit. J Pediatr (Rio J) [Internet]. 2015 Nov [acesso em 2017 Dez 29];91(6):535-42. Disponível em: http://www.ncbi.nlm.nih.gov/pubmed/26092227

7. Batalha MA, França AKTC, Conceição SIO, Santos AM, Silva FS, Padilha LL, et al. Processed and ultra-processed food consumption among children aged 13 to 35 months and associated factors. Cad Saúde Pública [Internet]. 2017 [acesso em 2017 Dez 29];33(11):16. Disponível em: http://www.scielo.br/pdf/csp/v33n11/1678-4464-csp33-11-e00152016.pdf

8. Barcelos GT, Rauber F, Vitolo MR. Produtos processados e ultraprocessados e ingestão de nutrientes em crianças. Ciência Saúde [Internet]. 2014 [acesso em 2017 Dec 29];7(3):155. Disponível em: http://revistaseletronicas. pucrs.br/ojs/index.php/faenfi/article/view/19755

9. Rauber F, Campagnolo PDB, Hoffman DJ, Vitolo MR. Consumption of ultra-processed food products and its effects on children's lipid profiles: a longitudinal study. Nutr Metab Cardiovasc Dis [Internet]. 2015 Jan [acesso em 2017 Dez 29];25(1):116-22. Disponível em: http://www.ncbi.nlm.nih.gov/pubmed/25240690

10. Louzada MLC, Martins APB, Canella DS, Baraldi LG, Levy RB, Claro RM, et al. Impact of ultra-processed foods on micronutrient content in the Brazilian diet. Rev Saude Publica [Internet]. 2015 [acesso em 2017 Dez 29];49:18. Disponível em: http://www.scielo.br/scielo.php?script=sci_arttext\&pid=S0034-89102015000100238\&lng=en\& tlng=en

11. Bielemann RM, Motta JV, Minten GC, Horta BL, Gigante DP. Consumption of ultra-processed foods and their 
impact on the diet of young adults. Rev Saúde Pública [Internet]. 2015 [acesso em 2017 Dez 29];49:28. Disponível em: https://www.ncbi.nlm.nih.gov/pubmed/26018785

12. Canella DS, Levy RB, Martins APB, Claro RM, Moubarac J-C, Baraldi LG, et al. Ultra-processed food products and obesity in brazilian households (2008-2009). PLoS One [Internet]. 2014 [acesso em 2018 Fev 4];9(3):e92752. Disponível em: http://www.ncbi.nlm.nih.gov/pubmed/24667658

13. Louzada MLC, Baraldi LG, Steele EM, Martins APB, Canella DS, Moubarac J-C, et al. Consumption of ultraprocessed foods and obesity in Brazilian adolescents and adults. Prev Med (Baltim) [Internet]. 2015 [acesso em 2018 Feb 4];81:9-15. Disponível em: http://www.ncbi.nlm.nih.gov/pubmed/26231112

14. Tavares LF, Fonseca SC, Garcia Rosa ML, Yokoo EM. Relationship between ultra-processed foods and metabolic syndrome in adolescents from a Brazilian Family Doctor Program. Public Health Nutr [Internet]. 2012 [acesso em2018 Fev 4];15(1):82-7. Disponível em: http://www.ncbi.nlm.nih.gov/pubmed/21752314

15. World Health Organization. WHO Child Growth Standards WHO Child Growth Standards Length/height-for-age, weight-for-age, weight-for-length, weight-for-height and body mass index-for-age Methods and development [Internet]. Genebra: WHO; 2006 [acesso em 2017 Dec 29]. Disponível em: http://www.who.int/childgrowth/ standards/Technical_report.pdf

16. Ministério da Saúde (BR), Secretaria de Atenção à Saúde, Departamento de Atenção Básica. Guia alimentar para a população brasileira [Internet]. Brasília: Ministério da Saúde; 2014 [acesso em 2017 Dez 29]. Disponível em: www.saude.gov.br/bvs

17. Malhi $P$, Venkatesh L, Bharti B, Singhi P. Feeding problems and nutrient intake in children with and without autism: a comparative study. Indian J Pediatr [Internet]. 2017 [acesso em 2017 Dez 29];84(4):283-8. Disponível em: http://www.ncbi.nlm.nih.gov/pubmed/28078576

18. Schreck KA, Williams K, Smith AF. A comparison of eating behaviors between children with and without autism. J Autism Dev Disord [Internet]. 2004 [acesso em 2017 Dez 29];34(4):433-8. Disponível em: http://www.ncbi.nIm. nih.gov/pubmed/15449518

19. Emond A, Emmett P, Steer C, Golding J. Feeding symptoms, dietary patterns, and growth in young children with autism spectrum disorders. Pediatrics [Internet]. 2010 [acesso em 2017 Dez 29];126(2):e337-42. Disponível em: http://www.ncbi.nlm.nih.gov/pubmed/20643716

20. Liu X, Liu J, Xiong X, Yang T, Hou N, Liang X, et al. Correlation between nutrition and symptoms: nutritional survey of children with autism spectrum disorder in Chongqing, China. Nutrients [Internet]. 2016 [acesso em 2017 Dez 29];8(5):E294. Disponível em: http://www.ncbi.nlm.nih.gov/pubmed/27187463

21. Ministério da Saúde (BR), Secretaria de Atenção à Saúde, Departamento de Atenção Básica. Dez passos para uma alimentação saudável: guia alimentar para crianças menores de dois anos: um guia para o profissional da saúde na atenção básica [Internet]. Brasília: Ministério da Saúde; 2015 [acesso em 2017 Dez 29]. Disponível em: http://bvsms.saude.gov.br/bvs/publicacoes/guia_dez_passos_alimentacao_saudavel_2ed.pdf

22. Ferreira NVR. Estado nutricional em crianças com transtorno do espectro autista. Curitiba: Universidade Federal do Paraná; 2016.

23. Bortolini GA, Gubert MB, Santos LMP. Consumo alimentar entre crianças brasileiras com idade de 6 a 59 meses. Cad Saúde Pública [Internet]. 2012 [acesso em 2017 Dez 29];28(9):1759-71. Disponível em http://www.scielo. br/scielo.php?script=sci_arttext\&pid=S0102-311X2012000900014\&Ing=pt\&tlng=pt

24. Schmitt L, Heiss CJ, Campbell EE. A Comparison of nutrient intake and eating behaviors of boys with and without autism. Top Clin Nutr [Internet]. 2008 [acesso em 2017 Dez 29];23(1):23-31. Disponível em: http://content. wkhealth.com/linkback/openurl?sid=WKPTLP:landingpage\&an=00008486-200801000-00003

25. Barcelos GT, Rauber F, Vitolo MR. Produtos processados e ultraprocessados e ingestão de nutrientes em crianças. Rev Ciência Saúde [Internet]. 2014 [acesso em 2018 Ago 21];7(3):155-61. Disponível em: https://www. researchgate.net/publication/277476324

26. Park MH, Falconer C, Viner RM, Kinra S. The impact of childhood obesity on morbidity and mortality in adulthood: a systematic review. Obes Rev [Internet]. 2012 [acesso em 2017 Dez 29];13(11):985-1000. Disponível em: http:// www.ncbi.nlm.nih.gov/pubmed/22731928 
27. Domingues G. Relação entre medicamentos e ganho de peso em indivíduos portadores de autismo e outras síndromes relacionadas [Internet]. Campo Grande; 2007 [acesso em 2017 Dez 29]. Disponível em: http://www. nutricaoativa.com.br/arquivos/monografia9.pdf

28. Hyman SL, Stewart PA, Schmidt B, Cain U, Lemcke N, Foley JT, et al. Nutrient intake from food in children with autism. Pediatrics [Internet]. 2012 [acesso em 2017 Dez 29];130(Suppl 2):S145-53. Disponível em: http://www. ncbi.nlm.nih.gov/pubmed/23118245

29. Caetano MV, Gurgel D. Perfil nutricional de crianças portadoras do transtorno do espectro autista. Rev Bras Promoç Saúde [Internet]. 2018 [acesso em 2018 Ago 20];31(1):1-11. Disponível em: http://periodicos.unifor.br/ RBPS/article/view/6714

30. Khogali Salih NA, Ibrahim NM, Ahmed LA. Nutritional status of autistic children and relationship with nutritional awareness of their mothers. Glob J Health Sci [Internet]. 2017 [acesso em 2017 Dez 29];9(7):177-85. Disponível em: http://www.ccsenet.org/journal/index.php/gjhs/article/view/68152

31. Ministério da Saúde (BR). Política Nacional de Alimentação e Nutrição [Internet]. Brasília: Ministério da Saúde; 2013 [acesso em 2018 Aug 20]. Disponível em: http://dab.saude.gov.br/portaldab/pnan.php

32. Malta DC, Morais OL Neto, Silva MMA, Rocha D, Castro AM, Reis AAC, et al. Política Nacional de Promoção da Saúde (PNPS): capítulos de uma caminhada ainda em construção. Ciênc Saúde Coletiva [Internet]. 2016 [acesso 2018 Aug 20];21(6):1683-94. Disponível em: http://www.scielo.br/scielo.php?script=sci_arttext\&pid=S1413$81232016000601683 \&$ lng $=$ pt\&tlng=pt

33. Gomes PTM, Lima LHL, Bueno MKG, Araújo LA, Souza NM. Autism in Brazil: a systematic review of family challenges and coping strategies. J Pediatr (Rio J) [Internet]. 2015 [acesso em 2018 Set 2];91(2):111-21. Disponível em: http://linkinghub.elsevier.com/retrieve/pii/S002175571400165X

34. Bandini LG, Anderson SE, Curtin C, Cermak S, Evans EW, Scampini R, et al. Food selectivity in children with autism spectrum disorders and typically developing children. J Pediatr [Internet]. 2010 [acesso em 2018 Set 2];157(2):259-64. Disponível em: http://dx.doi.org/10.1016/j.jpeds.2010.02.013

35. Sharp WG, Berry RC, McCracken C, Nuhu NN, Marvel E, Saulnier CA, et al. Feeding problems and nutrient intake in children with autism spectrum disorders: a meta-analysis and comprehensive review of the literature. J Autism Dev Disord. 2013;43(9):2159-73.

\section{Endereço do primeiro autor:}

Ana Karla de Araújo Almeida

Universidade CEUMA

Rua Josué Montello, 1

Bairro: Renascença II

CEP: 65075-120 - São Luís - MA - Brasil

E-mail: karllynha123@hotmail.com

\section{Endereço para correspondência:}

Carolina Abreu de Carvalho

Instituto Federal do Maranhão - IFMA Campus Barreirinhas

Rodovia MA-225, Km 04, Povoado Santa Cruz

CEP: 65590-000 - Barreirinhas - MA - Brasil

E-mail: carolcarvalho91@gmail.com 